\title{
Green's function and generalized phase shift for surface and interface problems
}

\author{
Roland E: Allen \\ Department of Physics, Texas A\&M University, College Station, Texas 77843
}

(Received 28 March 1977)

\begin{abstract}
For electrons, phonons, etc., and regardless of symmetry, the Green's function in any mixed WannierBloch representation is $G_{0}^{+}\left(z-z^{\prime}, \overline{\mathrm{k}} n \omega\right)=-i a \Sigma_{j} e^{i k_{j}}\left(z-z^{\prime}\right) / v\left(k_{j} \overline{\mathrm{k}} n\right) \operatorname{sgn}\left(z-z^{\prime}\right)+G_{\mathrm{BC}}$, where $\overline{\mathrm{k}}=\left(k_{x}, k_{y}\right), n$ is the branch index, and the values of $z$ correspond to lattice points. The $k_{j}$ are those values of $k_{z}$ for which the eigenvalue $\epsilon\left(k_{z} \bar{k} n\right)$ is equal to the parameter $\omega$, and for which $v\left(k_{j} \bar{k} n\right) \operatorname{sgn}\left(z-z^{\prime}\right)>0$, if $k_{j}$ is real, or $\operatorname{Im} k_{j} \operatorname{sgn}\left(z-z^{\prime}\right)>0$, if $k_{j}$ is complex. $G_{\mathrm{BC}}$ represents integrals around branch cuts, $a$ is the height of a unit cell, and $v\left(k_{z} \overline{\mathrm{k}} n\right) \equiv \partial \epsilon\left(k_{z} \overline{\mathrm{k}} n\right) / \partial k_{z}$. The above expression can be regarded as a generalization of the usual one-dimensional Green's function of quantum mechanics. $G_{0}^{+}(\omega)$ diverges whenever $\omega$ is such that some $v\left(k_{j} \overline{\mathrm{k}} n\right)$ goes to zero, and as a result the generalized phase shift $\eta(\omega \overline{\mathrm{k}})$ has discontinuities of $-\pi / 2$ at these values of $\omega$. These discontinuities are present regardless of the strength of $V$, the perturbation associated with creating a pair of surfaces or interfaces. There is an exception: If det $\vec{M}=0$, where $\overleftrightarrow{M}$ is a matrix defined in terms of the matrix elements of $V$, then the discontinuity is eliminated. This condition is analogous to that for a "zero-energy resonance" in $s$-wave potential scattering, and it will ordinarily occur only at particular transitional strengths of $V$. The condition is always satisfied for acoustic phonons at $\omega=\overline{\mathrm{k}}=0$, however, because of a restriction on the force constants. The significance of $\eta(\omega \overline{\mathrm{k}})$ is that the surface or interface density of states $\Delta \rho(\omega \overline{\mathrm{k}})$ is given by $\pi^{-1} \partial \eta(\omega \overline{\mathrm{k}}) / \partial \omega$. Each discontinuity of $-\pi / 2$ in $\eta(\omega \overline{\mathrm{k}})$ at an extremum $\omega_{0}$ thus produces a contribution $-\delta\left(\omega-\omega_{0}\right) / 2$ in $\Delta \rho(\omega \overline{\mathrm{k}})$.
\end{abstract}

\section{INTRODUCTION}

The purposes of this paper are (i) to obtain a form for the Green's function $G_{0}^{+}$which should be useful in treating surface and interface problems, and (ii) to prove that there are discontinuities of $-\frac{1}{2} \pi$ in the generalized phase shift ${ }^{1,2} \eta(\omega)$ at certain values of $\omega$. The significance of $\eta(\omega)$ is that it gives the change in the density of states when a pair of surfaces or interfaces is created, $\Delta \rho(\omega)$, through the relation ${ }^{2}$

$$
\Delta \rho(\omega)=\frac{1}{\pi} \frac{\partial \eta(\omega)}{\partial \omega}
$$

Thus a discontinuity of $-\frac{1}{2} \pi$ in $\eta(\omega)$ at some $\omega_{0}$ leads to a contribution $-\frac{1}{2} \delta\left(\omega-\omega_{0}\right)$ in $\Delta \rho(\omega)$.

The present treatment does not assume any symmetries (such as the existence of a reflection plane perpendicular to the $z$ axis, or time-reversal symmetry) other than translational invariance, and it is valid for any eigenvalue equation involving an Hermitian operator or matrix, such as the Schrödinger equation for electrons and the matrix eigenvalue equation for phonons.

In the following it is assumed that the eigenvalue $\epsilon\left(k_{z} \overline{\mathrm{k}} n\right)$ of Sec. II is an analytic function of the complex variable $k_{z}$ for all $k_{z}$ except branch points off the real axis. Kohn has shown that this is true for electrons ${ }^{3}$ and phonons ${ }^{4}$ in one dimension, and Blount ${ }^{5}$ and Krieger ${ }^{6}$ have extended the proof to three dimensions. There are some restrictions. For example, as Kohn points out, ${ }^{4}$ the behavior of the force constants in a metal implies that the phonon frequencies are not analytic for some real values of $k_{z}$.

It is also assumed that the matrix elements of the perturbation $V$ associated with creating a pair of surfaces or interfaces, the $V\left(k_{z} k_{z}^{\prime} n n^{\prime} \overline{\mathrm{k}}\right)$ of Sec. $\mathrm{V}$, are not infinite. This is analogous to the assumption in potential scattering theory that certain integrals involving the potential are not infi-. nite. (See Sec. VI.) The potential must fall off sufficiently fast as $r \rightarrow \infty$ and must not diverge too fast as $r \rightarrow 0$ in order for, e.g., Levinson's theorem to hold. ${ }^{\text {? }}$

We evaluate the Green's function in a mixed Wannier-Bloch representation. ${ }^{8-10}$ Such a representation is appropriate for surface and interface problems because the basis functions are localized in the $z$ direction, perpendicular to the surface or interface.

\section{UNPERTURBED CRYSTAL}

Consider a crystal with translational invariance in the $x, y$, and $z$ directions, for which some eigenvalue equation

$$
H_{0}|\overrightarrow{\mathrm{k}} n\rangle=\epsilon(\overrightarrow{\mathrm{k}} n)|\overrightarrow{\mathrm{k}} n\rangle
$$

holds. The $|\overrightarrow{\mathrm{k}} n\rangle$ are (generalized) Bloch functions, with $k_{x}$ and $k_{y}$ taken to be real but $k_{z}$ possibly complex, and $n$ is a branch index which distinguishes solutions with the same wave vector $\vec{k}$. It is assumed that the matrix or (possibly nonlocal) operator $H_{0}$ is Hermitian when the vectors or functions $|\overrightarrow{\mathrm{k}} n\rangle$ are restricted to those with real $k_{z}$. This implies that $\epsilon(\overrightarrow{\mathrm{k}} n)$ is real when $k_{z}$ is real. 
Let $a$ be the repeat distance for $H_{0}$ in the $z$ direction (i.e., the height of a "surface-adapted unit cell"). Because of the translational invariance of $H_{0}$, one can choose

$$
\begin{aligned}
& \epsilon\left(k_{z}+2 \pi / a, \overline{\mathrm{k}} n\right)=\epsilon\left(k_{z} \overline{\mathrm{k}} n\right), \\
& \left|k_{z}+2 \pi / a, \overline{\mathrm{k}} n\right\rangle=\left|k_{z} \overline{\mathrm{k}} n\right\rangle,
\end{aligned}
$$

where

$$
\overline{\mathrm{k}} \equiv\left(k_{x}, k_{y}\right),
$$

so all the distinct solutions to (2.1) are given by

$$
-\frac{\pi}{a}<\operatorname{Re} k_{z} \leqslant \frac{\pi}{a}
$$

Two representations will be used in the following: In the "Bloch representation" (or "crystal momentum representation"), the basis functions are the $\left|k_{z} \overline{\mathrm{k}} n\right\rangle$ for real $k_{z}$ which satisfy (2.5) and periodic boundary conditions, with $L$ being the periodicity length in the $z$ direction. In the "Wannier-Bloch representation," the basis functions are

$$
|z \overline{\mathrm{k}} n\rangle \equiv N^{-1 / 2} \sum_{k_{z}} e^{-i k_{z} z}\left|k_{z} \overline{\mathrm{k}} n\right\rangle,
$$

where

$$
N \equiv L / a
$$

and the summation includes all the real $k_{z}$ which satisfy (2.5) and the periodic boundary conditions. The values of $z$ are discrete and correspond to the positions of (surface-adapted) lattice points: $z=z\left(l_{3}\right)$, with $\overrightarrow{\mathrm{I}}=\left(l_{1}, l_{2}, l_{3}\right)$ labeling a lattice point.

The Green's function for the unperturbed crystal is, in the Wannier-Bloch representation,

$$
\begin{aligned}
& G_{0}\left(z z^{\prime}, \overline{\mathrm{k}} \overline{\mathrm{k}}^{\prime}, n n^{\prime}, \omega\right) \equiv\left\langle z \overline{\mathrm{k}} n\left|\left(\omega-H_{0}\right)^{-1}\right| z^{\prime} \overline{\mathrm{k}}^{\prime} n^{\prime}\right\rangle \\
&=\delta_{\overline{\mathrm{k}} \overline{\mathrm{k}}^{\prime}} \delta_{n n^{\prime}} G_{0}\left(z-z^{\prime}, \overline{\mathrm{k}} n \omega\right), \\
& G_{0}\left(z-z^{\prime}, \overline{\mathrm{k}} n \omega\right) \equiv N^{-1} \sum_{k_{z}} \frac{e^{i k_{z}\left(z-z^{\prime}\right)}}{\omega-\epsilon\left(k_{z} \overline{\mathrm{k}} n\right)} .
\end{aligned}
$$

For $\omega$ real, the "retarded" Green's function is

$$
G_{0}^{+}\left(z-z^{\prime}, \overline{\mathrm{k}} n \omega\right) \equiv G_{0}\left(z-z^{\prime}, \overline{\mathrm{k}} n, \omega+i \delta\right),
$$

with $\delta$ positive and $\delta \rightarrow 0$.

\section{EVALUATION OF GREEN'S FUNCTION}

After the recipe $\sum_{k_{g}} \rightarrow(L / 2 \pi) \int d k_{z}$ is used, (2.10) becomes

$$
G_{0}^{+}\left(z-z^{\prime}, \overline{\mathrm{k}} n \omega\right)=\frac{a}{2 \pi} \int_{-\pi / a}^{\pi / a} d k_{z} \frac{e^{i k_{z}\left(z-z^{\prime}\right)}}{\omega-\epsilon\left(k_{z} \mathrm{k} n\right)+i \delta} .
$$

We evaluate this integral by using the rectangular contour of Fig. 1, which is closed in the upper half-plane for $\operatorname{sgn}\left(z-z^{\prime}\right)=+1$ and in the lower halfplane for $\operatorname{sgn}\left(z-z^{\prime}\right)=-1$. The existence of branch points, and a possible choice of branch cuts, are indicated schematically in Fig. 1. In general, let $C^{\prime}$ represent curves around all the branch cuts (with each curve traversed in a positive direction, as illustrated in the figure), and let

$$
\begin{aligned}
G_{B C}\left(z-z^{\prime}, \overline{\mathrm{k}} n \omega\right) & \equiv-\operatorname{sgn}\left(z-z^{\prime}\right) \frac{a}{2 \pi} \\
& \times \int_{C^{\prime}} d k_{z} \frac{e^{i k_{\boldsymbol{z}}\left(z-z^{\prime}\right)}}{\omega-\epsilon\left(k_{z} \mathrm{k} n\right)+i \delta}, \quad \delta \rightarrow 0 .
\end{aligned}
$$

In the following, we assume that $G_{B C}$ does not diverge as $\omega \rightarrow \omega_{0}$, where $\omega_{0}$ is an extremal point defined by (4.1) and (4.7).

The contributions from the vertical-line segments of $C$ cancel because of (2.2) and the fact that $z-z^{\prime}=a \times$ integer. The contribution at infinity clearly vanishes for $z \neq z^{\prime}$. It also vanishes for $z=z^{\prime}$ if we now assume that $\left|\epsilon\left(k_{z} \overline{\mathrm{k}} n\right)\right| \rightarrow \infty$ as $\left|\operatorname{Im} k_{z}\right| \rightarrow \infty$ with $\operatorname{Re} k_{z}$ fixed ["almost everywhere" in the interval of (2.5)]. Then $G_{0}^{+}=G_{B C}$ $+2 \pi i \operatorname{sgn}\left(z-z^{\prime}\right) \times($ sum of residues at poles), with the $\operatorname{sgn}\left(z-z^{\prime}\right)$ factor coming from the fact that $C$ follows the real axis in a negative direction for $\operatorname{sgn}\left(z-z^{\prime}\right)=-1$, in Fig. 1(b). Whenever

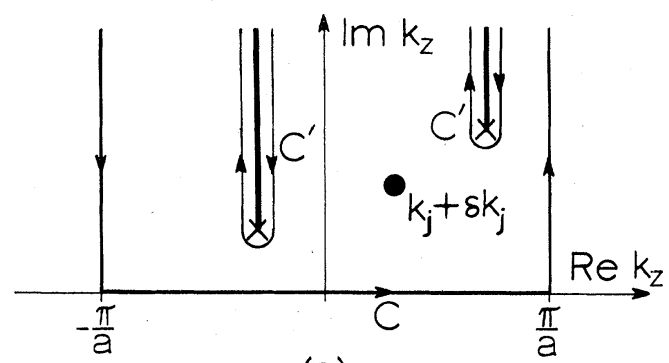

(a)

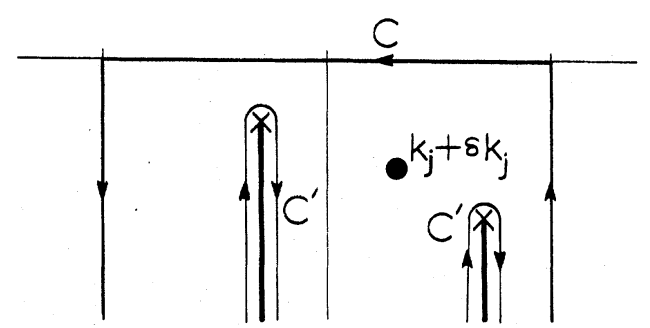

(b)

FIG. 1. (a) Contour $C$ used in evaluating (3.1) when $\operatorname{sgn}\left(z-z^{\prime}\right)=+1$. The vertical lines extend to infinity. The existence of branch points is indicated schematically. A representative pole $k_{j}+\delta k_{j}$ is shown. (b) Contour $C$ for $\operatorname{sgn}\left(z-z^{\prime}\right)=-1$. 


$$
\epsilon\left(k_{j}+\delta k_{j}, \overline{\mathrm{k}} n\right)=\omega+i \delta
$$

with $\delta k_{j} \rightarrow 0$ as $\delta \rightarrow 0$, there is a pole with residue

$$
\begin{aligned}
\frac{a}{2 \pi} \lim _{k_{z} \rightarrow k_{j}+6 k_{j}}\left[k_{z}-\left(k_{j}+\delta k_{j}\right)\right] & \frac{e^{i k_{z}\left(z-z^{\prime}\right)}}{\epsilon\left(k_{j}+\delta k_{j}, \mathrm{k} n\right)-\epsilon\left(k_{z} \mathrm{~K} n\right)} \\
= & -\frac{a}{2 \pi} \frac{e^{i\left(k_{j}+6 k_{j}\right)\left(z-z^{\prime}\right)}}{v\left(k_{j}+\delta k_{j}, \mathrm{~K} n\right)},
\end{aligned}
$$

where

$$
v\left(k_{z} \overline{\mathrm{k}} n\right) \equiv \frac{\partial \epsilon\left(k_{z} \overline{\mathrm{k}} n\right)}{\partial k_{z}} .
$$

We have assumed that $v \neq 0$ at the poles. The behavior of $G_{0}^{+}$as $v \rightarrow 0$ will be considered in Sec. IV.

As shown in Fig. $1, \operatorname{Im}\left(k_{j}+\delta k_{j}\right) \operatorname{sgn}\left(z-z^{\prime}\right)>0$. If $k_{j}$ is complex, no problems arise from just setting $\delta$ and $\delta k_{j}$ equal to zero in (3.3), so the requirement is just $\operatorname{Im} k_{j} \operatorname{sgn}\left(z-z^{\prime}\right)>0$. If $k_{j}$ is real, the expansion

$$
\epsilon\left(k_{j}+\delta k_{j}\right)=\epsilon\left(k_{j}\right)+v\left(k_{j}\right) \delta k_{j}+\cdots,
$$

in conjunction with (3.3), implies that $v\left(k_{j}\right) \operatorname{Im} \delta k_{j}$ $>0$. [Notice that $v\left(k_{j}\right)$ is real for $k_{j}$ real, since $\epsilon\left(k_{z}\right)$ is real for $k_{z}$ real.] This requirement, plus the one in the first sentence of this paragraph, then implies that $v\left(k_{j}\right) \operatorname{sgn}\left(z-z^{\prime}\right)>0$.

Combining the above results, we get

$$
\begin{aligned}
G_{0}^{+}\left(z-z^{\prime}, \overline{\mathrm{k}} n \omega\right)= & -i a \sum_{j} \frac{e^{i k_{j}\left(z-z^{\prime}\right)}}{v\left(k_{j} \mathrm{k} n\right)} \operatorname{sgn}\left(z-z^{\prime}\right) \\
& +G_{B C}\left(z-z^{\prime}, \overline{\mathrm{k}} n \omega\right)
\end{aligned}
$$

where the $k_{j}$ are those values of $k_{z}$ satisfying

$$
\epsilon\left(k_{j} \overline{\mathrm{k}} n\right)=\omega,
$$

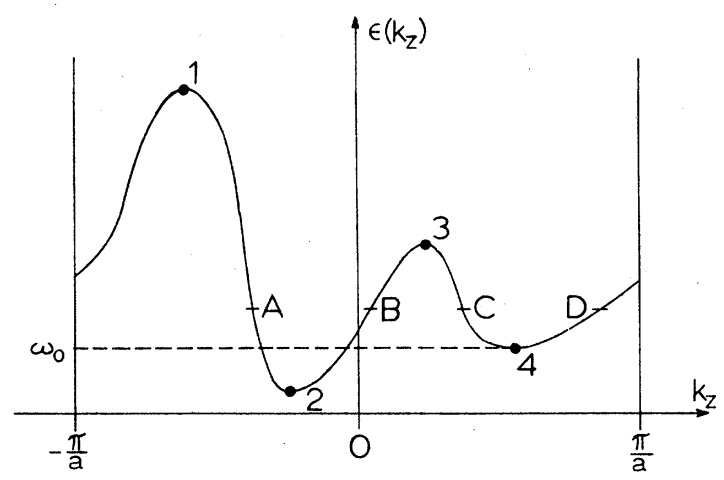

FIG. 2. Representative graph of eigenvalue $\epsilon\left(k_{z} \bar{k} n\right)$ vs real wave vector $k_{z}$, for a fixed planar wave vector $\mathrm{k}$ and a given branch labeled by $n$. The four numbered points are extrema where $v\left(k_{z}\right)=0$. As $\omega$ increases from just below an extremum $\omega_{0}$ to just above, $\eta(\omega)$ usually changes discontinuously by $-\frac{1}{2} \pi$. The four lettered points illustrate that, at fixed $\epsilon$, there are as many solutions with $v\left(k_{z}\right)$ negative $\left(A\right.$ and $C$ ) as with $v\left(k_{z}\right)$ positive $(B$ and $D)$. and for which

$$
\begin{aligned}
& v\left(k_{j} \overline{\mathrm{k}} n\right) \operatorname{sgn}\left(z-z^{\prime}\right)>0, \quad k_{j} \text { real }, \\
& \operatorname{Im} k_{j} \operatorname{sgn}\left(z-z^{\prime}\right)>0, \quad k_{j} \text { complex. }
\end{aligned}
$$

Exactly half the solutions to (3.8) also satisfy (3.9), regardless of symmetry: If $k_{z}$ is complex, then Schwarz's reflection principle ${ }^{11}$ requires that $\epsilon\left(k_{z}^{*} \overline{\mathrm{k}} n\right)=\epsilon^{*}\left(k_{z} \overline{\mathrm{k}} n\right)$. For every $k_{z}$ corresponding to a real eigenvalue $\epsilon\left(k_{z}\right)$, therefore, there is a $k_{z}^{*}$ corresponding to the same eigenvalue. I.e., for every evanescent wave decaying toward the right and satisfying (3.8), there is another decaying toward the left. If $k_{z}$ is real, (2.2) implies that there is some $k_{z}^{\prime}$ such that $\epsilon\left(k_{z}^{\prime}\right)=\epsilon\left(k_{z}\right)$ and $v\left(k_{z}^{\prime}\right)$ is opposite in sign to $v\left(k_{z}\right)$, as illustrated in Fig. 2. I.e., for every wave propagating to the right, and satisfying (3.8), there is another propagating to the left.

\section{BEHAVIOR OF GREEN'S FUNCTION AT EXTREMA}

Let $k_{0}$ represent an extremum in $\epsilon\left(k_{z} \overline{\mathrm{k}} n_{0}\right)$ for some branch $n_{0}$ at a fixed $\overline{\mathrm{k}}$,

$$
v\left(k_{0} \overline{\mathrm{k}} n_{0}\right)=0, \epsilon\left(k_{0} \overline{\mathrm{k}} n_{0}\right) \text { real . }
$$

For nearby $k_{z}$,

$$
\begin{aligned}
& \epsilon\left(k_{z} \overline{\mathrm{k}} n_{0}\right)-\epsilon\left(k_{0} \overline{\mathrm{k}} n_{0}\right) \simeq\left(k_{z}-k_{0}\right)^{2} / 2 m^{*}, \\
& v\left(k_{z} \overline{\mathrm{k}} n_{0}\right) \simeq\left(k_{z}-k_{0}\right) / m^{*}, \\
& \frac{1}{m^{*}} \equiv\left(\frac{\partial^{2} \epsilon\left(k_{z} \overline{\mathrm{k}} n_{0}\right)}{\partial k_{z}^{2}}\right)_{k_{z}=k_{0}} .
\end{aligned}
$$

We assume that $k_{0}$ must be real, since, according to Heine, ${ }^{12}$ it is "vanishingly probable" for (4.1) to hold if $k_{0}$ is not real. We also assume that $m^{*}$ is finite-i.e., that the second derivative $\partial^{2} \epsilon / \partial k_{s}^{2}$ is nonzero at those points where the first derivative vanishes.

Since $k_{0}$ is real, we can choose $k_{z}$, and thus $\epsilon\left(k_{z}\right)$, to be real in evaluating $m^{*}$ according to (4.4), so $m^{*}$ is real. As $\omega \rightarrow \epsilon\left(k_{0} \overline{\mathrm{k}} n_{0}\right)$, the dominant $k_{j}$ in (3.7) is

$$
\tilde{k} \simeq k_{0}+\Delta k \operatorname{sgn}\left(z-z^{\prime}\right),
$$

where

$$
\begin{aligned}
& (\Delta k)^{2} \equiv 2 m *\left(\omega-\omega_{0}\right), \\
& \omega_{0} \equiv \epsilon\left(k_{0} \overline{\mathrm{k}} n_{0}\right),
\end{aligned}
$$

according to (3.8) and (4.2). The sign of $\Delta k$ is determined by (3.9) and (4.3): At a minimum (along the real axis), $m^{*}$ is positive and

$$
\Delta k= \begin{cases}i\left[2 m^{*}\left(\omega_{0}-\omega\right)\right]^{1 / 2}, & \omega<\omega_{0}, \\ {\left[2 m^{*}\left(\omega-\omega_{0}\right)\right]^{1 / 2},} & \omega>\omega_{0} .\end{cases}
$$

At a maximum, $m^{*}$ is negative and 


$$
\Delta k= \begin{cases}-\left[2 m^{*}\left(\omega-\omega_{0}\right)\right]^{1 / 2}, & \omega<\omega_{0} \\ i\left[2 m^{*}\left(\omega_{0}-\omega\right)\right]^{1 / 2}, & \omega>\omega_{0} .\end{cases}
$$

The positive root is to be taken in (4.8) and (4.9). Thus,

$$
\operatorname{disc} \operatorname{Arg} \Delta k=-\frac{1}{2} \pi,
$$

regardless of whether this point is a minimum or a maximum along the real axis, where $\operatorname{disc} A(\omega)$ is defined to be the discontinuous change in a quantity $A(\omega)$ as $\omega$ increases through $\omega_{0}$.

There is a point worth mentioning: From (4.8) and (4.9) alone, it is not clear whether we should take the change in $\operatorname{Arg} \Delta k$ to be $-\frac{1}{2} \pi$ or $-\frac{1}{2} \pi+2 \pi$ $x$ integer. This ambiguity is resolved by recalling that $\mathrm{a}+i \delta$ should really be added to $\omega$ to obtain $G_{0}^{+}(\omega)$, which implies, according to the first sentence of the paragraph containing (3.6), that a $+i|\delta k|$ should really be added to $\Delta k$. We should really let $\delta$ and $\delta k \rightarrow 0$ only after determining the phase shift. This point is made by de $\mathrm{Witt}^{1}$ for the case of bound states on his p. 1567: As $\omega$ increases from $-\infty$, the addition of $a+i \delta$ to $\omega$ requires that we pass around the bound state energy $\omega_{b}$ in the upper half-plane, so that $a-\pi$ is added to the phase of $\operatorname{det}\left[\overrightarrow{1}-\ddot{G}_{0}^{+}(\omega) \cdot \vec{V}\right]$, and $a+\pi$ to $\eta(\omega)$. In the present case, suppose first that $m^{*}<0$, so that (4.9) applies. Then, as $\omega$ increases to $\omega_{0}, \Delta k+i|\delta k|$ moves along just above the negative real axis until it reaches the imaginary axis, and $\operatorname{Arg}(\Delta k+i|\delta k|)$ changes continuously by $-\frac{1}{2} \pi$. If $m^{*}>0$, (4.8) applies, and as $\omega$ increases past $\omega_{0}, \Delta k+i|\delta k|$ moves away from the imaginary axis just above the positive real axis, so that $\operatorname{Arg}(\Delta k+i|\delta k|)$ again changes continuously by $-\frac{1}{2} \pi$. In the limit $\delta \rightarrow 0, \eta(\omega)$ changes discontinuously by $+\pi$ when $\omega$ increases through some $\omega_{b}$. In the limit $\delta k \rightarrow 0, \operatorname{Arg} \Delta k$ changes discontinuously by $-\frac{1}{2} \pi$ when $\omega$ increases through some $\omega_{0}$. But for $\delta \neq 0$, the phase of $\operatorname{det}\left[\overrightarrow{1}-\bar{G}_{0}(\omega\right.$ $+i \delta) \cdot \overline{\mathrm{V}}]$ changes continuously in the first case, and for $\delta k \neq 0$, the phase of $\Delta k+i|\delta k|$ changes continuously in the second case.

According to (4.3) and (4.5),

$$
v\left(\tilde{k} \overline{\mathrm{k}} n_{0}\right) \simeq \Delta k \operatorname{sgn}\left(z-z^{\prime}\right) / m^{*},
$$

so (3.7) implies that

$$
\begin{aligned}
G_{0}^{+}\left(z-z^{\prime}, \overline{\mathrm{k}} n_{0} \omega\right)= & -i a m^{*} e^{i k_{0}\left(z-z^{\prime}\right)} \frac{e^{i \Delta k\left|z-z^{\prime}\right|}}{\Delta k} \\
& -i a \sum_{j}^{\prime} \frac{e^{i k_{j}\left(z-z^{\prime}\right)}}{v\left(k_{j} \mathrm{k} n_{0}\right)} \operatorname{sgn}\left(z-z^{\prime}\right) \\
& +G_{B C}\left(z-z^{\prime}, \overline{\mathrm{k}} n_{0} \omega\right),
\end{aligned}
$$

where the primed summation excludes the value $k_{j}=\tilde{k}$.

Eq. (4.12) [or, more generally, (3.7)] is a generalization of the usual one-dimensional Green's function for particles of mass $m$ in quantum mechanics, which can be obtained as a special case by suppressing the branch index $n_{0}$, the planar wave vector $\overline{\mathrm{k}}$, the primed summation over $j$, the contribution $G_{B C}$ from branch cuts, and $a$, and letting $m^{*} \rightarrow m, k_{0} \rightarrow 0$, and $\Delta k \rightarrow k$.

\section{DISCONTINUITIES IN THE GENERALIZED PHASE SHIFT}

Starting with the unperturbed crystal of the preceeding sections, we break the translational invariance in the $z$ direction by creating a pair of surfaces or interfaces, and we represent the new matrix or operator in the perturbed crystal by

$$
H=H_{0}+V \text {. }
$$

The new eigenvalue equation is

$$
H|i \overline{\mathrm{k}}\rangle=\epsilon(i \overline{\mathrm{k}})|i \overline{\mathrm{k}}\rangle,
$$

where $i$ distinguishes solutions with the same $\overline{\mathrm{k}}$.

We assume that $V$ can be represented in terms of the Bloch functions which satisfy the periodic boundary conditions of Sec. II. This requires (as a mathematical device) that $V$ satisfy these same boundary conditions, i.e., that

$$
V(z+L, \overline{\mathrm{r}})=V(z \overline{\mathrm{r}})
$$

if $V$ is local, or

$$
V\left(z+L, \overline{\mathrm{r}} ; z^{\prime}+L, \overline{\mathrm{r}}^{\prime}\right)=V\left(z \overline{\mathrm{r}} ; z^{\prime} \overline{\mathrm{r}}^{\prime}\right)
$$

if $V$ is nonlocal, where

$$
\overline{\mathrm{r}} \equiv(x, y) \text {. }
$$

$V$ corresponds to creating interfaces between two materials $A$ and $B$ at two planes $z=0$ and $z=z_{0}$, starting with only material $A$. The perturbed crystal has material $A$ between $z=0$ and $z=z_{0}$, material $B$ between $z=z_{0}$ and $z=L$, material $A$ between $z=L$ and $z=L+z_{0}$, etc. If free surfaces are created, then material $B$ is vacuum. It is, of course, impossible to create a single interface, and $V$ always corresponds to complementary interfaces at $z=0$ and $z=z_{0}$. If we do not want the interfaces to interfere, we choose $z_{0} \rightarrow \infty$ and $L-z_{0}$ $\rightarrow \infty$, as well as $L \rightarrow \infty$.

The generalized phase shift resulting from the perturbation $V$ is $^{1,2}$

$$
\eta(\omega \overline{\mathrm{k}}) \equiv-\operatorname{Arg} \operatorname{det}\left[\overrightarrow{\mathrm{I}}-\overline{\mathrm{G}}_{0}^{+}(\overline{\mathrm{k}} \omega) \cdot \overrightarrow{\mathrm{V}}(\overline{\mathrm{k}})\right] .
$$

In the Wannier-Bloch representation, the elements of $\overrightarrow{\mathrm{G}}_{0}^{+}$and $\overrightarrow{\mathrm{V}}$ are labeled by $z, n$ and $z^{\prime}, n^{\prime}$. In the Bloch representation, the elements are labeled by $k_{z}, n$ and $k_{z}^{\prime}, n^{\prime}$.

As $\omega \rightarrow \omega_{0}$, all the elements $G_{0}^{+}\left(z-z^{\prime}, \overline{\mathrm{k}} n_{0} \omega\right)$ in the Wannier-Bloch representation diverge as $1 / \Delta k$, according to (4.12). Since there are $N$ values of $z$ it may then appear that a $(1 / \Delta k)^{N}$ can be factored out 
of the determinant of (5.6). However, cancellations within a determinant can cause it to diverge more slowly than may first seem the case. For example,

$$
\left|\begin{array}{cc}
\frac{1}{x} & \frac{1}{x}-1 \\
\frac{1}{x}-1 & \frac{1}{x}
\end{array}\right|
$$

diverges as $1 / x$, instead of $1 / x^{2}$, as $x \rightarrow 0$. By switching to the Bloch representation, we now show that the determinant of (5.6) diverges as $1 / \Delta k$, instead of $(1 / \Delta k)^{N}$.

In the Bloch representation,

$$
\begin{aligned}
G_{0}^{+}\left(k_{z} k_{z}^{\prime}, \overline{\mathrm{k}} \overline{\mathrm{k}}^{\prime}, n n^{\prime}, \omega\right)= & G_{0}^{+}\left(k_{z} k_{z}^{\prime}, \overline{\mathrm{k}} n \omega\right) \\
& \times \delta_{\overline{\mathrm{k}} \overline{\mathrm{k}}^{\prime}} \delta_{n n^{\prime}}
\end{aligned}
$$

after (2.6) and (2.9) are used. The periodic boundary conditions require that

$$
\sum_{z} e^{i\left(k_{z}-k_{z}^{\prime}\right) z}=N \delta_{k_{z} k_{z}^{\prime}}
$$

so

$$
\begin{aligned}
& G_{0}^{+}\left(k_{z} k_{z}^{\prime}, \overline{\mathrm{k}} n \omega\right)= N^{-1} \sum_{z^{\prime}} e^{i\left(k_{z}^{\prime}-k_{z}\right) z^{\prime}} \\
& \times \sum_{z-z^{\prime}} G_{0}^{+}\left(z-z^{\prime}, \overline{\mathrm{k}} n \omega\right) e^{-i k_{z}\left(z-z^{\prime}\right)} \\
&=\delta_{k_{z} k_{z}^{\prime}} G_{0}^{+}\left(k_{z} \overline{\mathrm{k}} n \omega\right) \\
& G_{0}^{+}\left(k_{z} \overline{\mathrm{k}} n \omega\right) \equiv \sum_{z=z^{\prime}} e^{-i k_{z}\left(z-z^{\prime}\right)} G_{0}^{+}\left(z-z^{\prime}, \overline{\mathrm{k}} n \omega\right)
\end{aligned}
$$

Let $G^{\prime}\left(z-z^{\prime}, \overline{\mathrm{k}} n \omega\right)$ represent that part of $G_{0}^{+}\left(z-z^{\prime}\right.$, $\overline{\mathrm{k}} n \omega)$ that remains finite as $\omega \rightarrow \omega_{0}$. According to (4.12),

$$
\begin{aligned}
G_{0}^{+}\left(z-z^{\prime}, \overline{\mathrm{k}} n \omega\right)= & -i a m^{*} e^{i k_{0}\left(z-z^{\prime}\right)} \frac{e^{i \Delta k\left|z-z^{\prime}\right|}}{\Delta k} \delta_{n n_{0}} \\
& +G^{\prime}\left(z-z^{\prime}, \overline{\mathrm{k}} n \omega\right) .
\end{aligned}
$$

Beyond this point the argument becomes awkward unless $k_{0}$ is one of the values of $k_{z}$ allowed by the periodic boundary conditions. Since this has zero probability for a given dispersion relation, we resort to the artifice of perturbing $H_{0}$ (once $\overline{\mathrm{k}}, n_{0}$, and $L$ have been specified) in such a way that the extremum in the dispersion relation is shifted from $k_{0}$ to $k_{0}^{\prime}$, the value of $k_{z}$ that is closest to $k_{0}$ of the $N$ values allowed by the periodic boundary conditions. Since $\left|k_{0}^{\prime}-k_{0}\right|<2 \pi / L$, the new $H_{0}, G_{0}^{+}$, and $\eta(\omega \overline{\mathrm{k}})$ are indistinguishable from the old as $L \rightarrow \infty$. In the following, we rename $k_{0}^{\prime}$ and call it $k_{0}$.

$$
\text { As } \Delta k \rightarrow 0, e^{i \Delta k\left|z-z^{\prime}\right|} \rightarrow 1 \text { and (5.13) gives }
$$

$$
\begin{aligned}
G_{0}^{+}\left(k_{z} \overline{\mathrm{k}} n \omega\right)=- & \frac{i a m^{*}}{\Delta k} \sum_{z} e^{i\left(k_{0}-k_{z}\right) z} \delta_{n n_{0}} \\
& +\sum_{z} e^{-i k_{z} z} G^{\prime}(z \overline{\mathrm{k}} n \omega) \\
& =-\frac{i m^{*} L}{\Delta k} \delta_{k_{z} k_{0}} \delta_{n n_{0}}+G^{\prime}\left(k_{z} \overline{\mathrm{k}} n \omega\right),
\end{aligned}
$$

in view of (5.10). Thus, in the Bloch representation, $\overrightarrow{\mathrm{G}}_{0}^{+}$is diagonal and only the one element labeled by $k_{0}$ and $n_{0}$ diverges.

Consider the expression (5.6) for $\eta(\omega \overline{\mathrm{k}})$ : Since $\ddot{\mathrm{G}}_{0}^{+}$is diagonal,

$$
\begin{aligned}
& {\left[\overline{\mathrm{I}}-\ddot{\mathrm{G}}_{0}^{+}(\overline{\mathrm{k}} \omega) \cdot \overrightarrow{\mathrm{V}}(\overline{\mathrm{k}})\right]_{k_{z^{n}, k_{z}^{\prime} n^{\prime}}}} \\
& \quad=\delta_{k_{z} k_{z}^{\prime}} \delta_{n n^{\prime}}-G_{0}^{+}\left(k_{z} \overline{\mathrm{k}} n \omega\right) V\left(k_{z} k_{z}^{\prime} n n^{\prime} \overline{\mathrm{k}}\right) .
\end{aligned}
$$

Then, according to (5.16),

$$
\begin{array}{r}
\operatorname{det}\left[\overrightarrow{\mathrm{l}}-\ddot{\mathrm{G}}_{0}^{+}(\overline{\mathrm{k}} \omega) \cdot \overrightarrow{\mathrm{V}}(\overline{\mathrm{k}})\right]-\frac{1}{\Delta k} \operatorname{det} \overrightarrow{\mathrm{M}}(\omega \overline{\mathrm{k}}) \\
\text { as } \omega \rightarrow \omega_{0},
\end{array}
$$

where

$$
\begin{array}{r}
M\left(k_{z} n k_{z}^{\prime} n^{\prime} ; \omega \overline{\mathrm{k}}\right) \equiv \delta_{k_{z} k_{z}^{\prime}} \delta_{n n^{\prime}}-G_{0}^{+}\left(k_{z} \overline{\mathrm{k}} n \omega\right) V\left(k_{z} k_{z}^{\prime} n n^{\prime} \overline{\mathrm{k}}\right), \\
k_{z} \neq k_{0} \text { or } n \neq n_{0}(5.19 \mathrm{a}) \\
\equiv i m^{*} L V\left(k_{0} k_{z}^{\prime} n_{0} n^{\prime} \overline{\mathrm{k}}\right), \\
k_{z}=k_{0} \text { and } n=n_{0} \cdot(5.19 \mathrm{~b})
\end{array}
$$

We are temporarily assuming that at least one of the $V\left(k_{0} k_{z}^{\prime} n_{0} n^{\prime} \overline{\mathrm{k}}\right)$ is nonzero. Then

$$
\begin{aligned}
\eta(\omega \overline{\mathrm{K}}) & =-\operatorname{Arg}(1 / \Delta k) \operatorname{det} \overline{\mathrm{M}}(\omega \overline{\mathrm{K}})] \\
& =\operatorname{Arg} \Delta k-\operatorname{Arg} \operatorname{det} \overline{\mathrm{M}}(\omega \overline{\mathrm{K}})
\end{aligned}
$$

in the limit $\omega \rightarrow \omega_{0}$. If $\operatorname{det} \overline{\mathrm{M}}\left(\omega_{0} \overline{\mathrm{k}}\right) \neq 0,(4.10)$ then implies that

$$
\operatorname{disc} \eta(\omega \overline{\mathrm{k}})=-\frac{1}{2} \pi
$$

as $\omega$ increases through any $\omega_{0}$ defined by (4.7) and (4.1).

\section{GENERALIZATIONS: MORE THAN ONE EXTREMUM$$
\operatorname{AT} \omega_{0} \text { OR } \operatorname{det} \overleftrightarrow{M}\left(\omega_{0}\right)=0
$$

In the foregoing, it was tacitly assumed that at $\omega_{0}$ there is an extremum for only one band $n_{0}$ at only one wave vector $k_{0}$. We now generalize to the case that there are $N_{0}$ extrema at $\omega_{0}$, so that (4.1), (4.7), and (4.4) become

$$
\begin{aligned}
& v\left(k_{0 i} \overline{\mathrm{k}} n_{0 i}\right)=0, \quad i=1,2, \ldots, N_{0}, \\
& \epsilon\left(k_{0 i} \overline{\mathrm{k}} n_{0 i}\right)=\omega_{0}, \omega_{0} \text { real }, \\
& \frac{1}{m_{i}^{*}} \equiv\left(\frac{\partial^{2} \epsilon\left(k_{z} \overline{\mathrm{k}} n_{0 i}\right)}{\partial k_{z}^{2}}\right) k_{z}=k_{0 i} .
\end{aligned}
$$


After generalization of the various steps, (5.16) becomes

$$
G_{0}^{+}\left(k_{z} \overline{\mathrm{k}} n \omega\right)=-i L \sum_{i=1}^{N_{0}} \frac{m_{i}^{*}}{\Delta k_{i}} \delta_{k_{z} k_{0} i} \delta_{n n_{0 i}}+G^{\prime}\left(k_{z} \overline{\mathrm{k}} n \omega\right),
$$

where $\Delta k_{i}$ is defined by (4.8) or (4.9) with $m^{*} \rightarrow m_{i}^{*}$. Then (5.18) and (5.19) are changed to

$$
\begin{aligned}
\operatorname{det}\left[\overrightarrow{\mathrm{l}}-\ddot{\mathrm{G}}_{0}^{+}(\overline{\mathrm{k}} \omega) \cdot \overrightarrow{\mathrm{V}}(\overline{\mathrm{k}})\right]-\left(\prod_{i=1}^{N_{0}} \frac{1}{\Delta k_{i}}\right) \operatorname{det} \overline{\mathrm{M}}(\omega \overline{\mathrm{k}}) \\
\text { as } \omega \rightarrow \omega_{0} \\
\begin{aligned}
M\left(k_{z} n k_{z}^{\prime} n^{\prime} ; \omega \overline{\mathrm{k}}\right) \equiv & i m_{i}^{*} L V\left(k_{0 i} k_{z}^{\prime} n_{0 i} n^{\prime} \overline{\mathrm{k}}\right) \\
n=n_{0 i} \text { and } k_{z}=k_{0 i} & \\
= & \delta_{k_{z} k_{z}^{\prime}} \delta_{n n^{\prime}}-G_{0}^{+}\left(k_{z} \overline{\mathrm{k}} n \omega\right) \\
& \times V\left(k_{z} k_{z}^{\prime} n n^{\prime} \overline{\mathrm{k}}\right) \text { otherwise }
\end{aligned}
\end{aligned}
$$

I.e., $N_{0}$ rows of $\ddot{\mathrm{M}}$ are different from those of $\overline{\mathrm{I}}-\overrightarrow{\mathrm{G}}_{0}^{+} \cdot \overrightarrow{\mathrm{V}}$. Finally, (5.21) becomes

$$
\eta(\omega \overline{\mathrm{k}})=\sum_{i=1}^{N_{\mathrm{Q}}} \operatorname{Arg} \Delta k_{i}-\operatorname{Arg} \operatorname{det} \overline{\mathrm{M}}(\omega \overline{\mathrm{k}})
$$

in the limit $\omega \rightarrow \omega_{0}$, and (4.10) implies that

$$
\operatorname{disc} \eta(\omega \overline{\mathrm{k}})=-N_{0} \frac{1}{2} \pi
$$

as $\omega$ increases through $\omega_{0}$.

It was also assumed that $\operatorname{det} \overline{\mathrm{M}}\left(\omega_{0} \overline{\mathrm{K}}\right) \neq 0$. Suppose that

$$
\operatorname{det} \ddot{\mathrm{M}}(\omega \overline{\mathrm{K}}) \propto(\Delta k)^{M_{0}} \text { as } \omega \rightarrow \omega_{0},
$$

where $\Delta k$ is any one of the $\Delta k_{i}$ in the general case discussed just above. [According to (4.8) and (4.9), the $\Delta k_{i}$ differ only by multiplicative constants.]

Then (6.5) implies that

$$
\operatorname{disc} \eta(\omega \overline{\mathrm{k}})=\left(M_{0}-N_{0}\right) \frac{1}{2} \pi
$$

as $\omega$ increases through $\omega_{0}$. For example, if there were a simple pole in $\operatorname{det} \ddot{\mathrm{M}}, M_{0}=-1$, we would have a discontinuous change in $\eta$ of $-\pi$ (for $N_{0}=1$ ), i.e., the usual $-\frac{1}{2} \pi$ plus an extra $-\frac{1}{2} \pi$ from the pole in det $\ddot{M}$. However, our assumption that the matrix elements of $V$ are not infinite implies that the same is true of $\operatorname{det} \ddot{M}$. There is an analogy in $s$-wave potential scattering: For potentials $V(r)$ satisfying ${ }^{7}$

$$
\int_{0}^{\infty} d r r|V(r)|<\infty, \quad \int_{0}^{\infty} d r r^{2}|V(r)|<\infty,
$$

the Jost function $f(k)$ is well behaved at $k=0$; but for model potentials violating these restrictions, $f(k)$ can have a pole at $k=0$, and a simple pole leads to a discontinuity of $-\frac{1}{2} \pi$ in the phase shift at $k=0 .{ }^{13}$
Instead of $\operatorname{det} \bar{M}(\omega \overline{\mathrm{K}}) \rightarrow 0$ as $\omega \rightarrow \omega_{0}$, we can have $\operatorname{det} \overline{\mathrm{M}}(\omega \overline{\mathrm{K}})=0$ for all $\omega$ if $M_{0}$ of the rows defined in (6.4a) are identically zero, with $M_{0} \leqslant N_{0}$. Then, as can be seen from (5.17), $M_{0}$ factors of $1 / \Delta k_{i}$ are removed from (6.3), and only $N_{0}-M_{0}$ factors remain. It follows that (6.8) also holds in this case, although for a different reason. In either case, if $M_{0}=N_{0}$, then (6.8) implies that

$$
\operatorname{disc} \eta(\omega \overline{\mathrm{k}})=0
$$

i.e., the discontinuity is eliminated. This exceptional case (or any case when $M_{0} \neq 0$ ) is analogous to a "zero-energy resonance" in $s$-wave potential scattering, for which the Jost function $f(0)$ equals zero and an exceptional version of Levinson's theorem holds. ${ }^{14}$ In $s$-wave potential scattering, a discontinuity of $+\frac{1}{2} \pi$ is added to the usual discontinuity of 0 when $f(0)=0$. In the present case (for $M_{0}=N_{0}=1$ ), a discontinuity of $+\frac{1}{2} \pi$ is added to the usual discontinuity of $-\frac{1}{2} \pi$ when $\operatorname{det} \overline{\mathrm{M}}\left(\omega_{0}\right)=0$.

A trivial example of $(6.10)$ holds when the perturbation $V$ is identically zero, so that all of the $N_{0}$ rows defined in (6.4a) contain zeros. In this case, of course, the phase shift can be taken to be identically zero, according to (5.6), and a discontinuity is impossible.

Whenever $V$ produces a discrete "bound" state at an energy $\omega_{b}$, there is a discontinuity of $+\pi$ in $\eta(\omega \overline{\mathrm{k}})$ at $\omega=\omega_{b}$, according to (1.1). When (6.10) holds (for $N_{0}=1$ ), therefore, it is as if $V$ is just strong enough to produce "half a bound state" at $\omega=\omega_{0}$, which gives a discontinuity of $+\frac{1}{2} \pi$ and thus cancels the usual discontinuity of $-\frac{1}{2} \pi$. The usual discontinuity, on the other hand, implies that a general perturbation $V$, no matter how weak, ordinarily "destroys half a state" at every eigenvalue $\omega_{0}$ where there is an extremum in the dispersion relation $\epsilon\left(k_{z} \overline{\mathrm{k}} n\right)$ versus $k_{z}$.

\section{ABSENCE OF DISCONTINUITY FOR ACOUSTIC PHONONS AT $\omega=\overline{\mathrm{k}}=0$}

We expect that (6.10) will ordinarily have "zero probability," and will hold only when the perturbation has exactly the right transitional strength. This is the case for a zero-energy resonance in $s$ wave potential scattering, which occurs only when the parameters in a model potential have exactly the right values. However, as we show below, (6.10) always holds for acoustic phonons at $\omega=$ $\overline{\mathrm{k}}=0$, because of a condition on the force constants. This corresponds to the fact that the three "uniform translation modes," at zero frequency, are present in both the perturbed and unperturbed crystals. If there were a discontinuity of $-3 \pi / 2$ in $\eta(\omega \overline{\mathrm{k}})$ at $\omega=\overline{\mathrm{k}}=0$, then (1.1) implies that each of the uniform translation modes would be "half 
destroyed" by the creation of a pair of surfaces or interfaces.

For phonons, we introduce a third set of basis functions $|\overrightarrow{\mathbf{r}} \kappa\rangle$, each of which is localized entirely on an atom labeled by $\kappa$ in a unit cell whose lattice point is at $\overrightarrow{\mathrm{r}}=(\overline{\mathrm{r}}, z)$. We remark that $|\overrightarrow{\mathrm{r}} \kappa\rangle,\left|k_{z} \overline{\mathrm{k}} n\right\rangle$, and $|z \overline{\mathrm{k}} n\rangle$ are all three-dimensional vectors (with $x, y$, and $z$ components). In the $|\overrightarrow{\mathrm{r}} \kappa\rangle$ representation, (2.1) becomes

$$
\begin{aligned}
\sum_{\overrightarrow{\mathrm{r}}^{\prime} \kappa^{\prime}}\left\langle\overrightarrow{\mathrm{r}} \kappa\left|H_{0}\right| \overrightarrow{\mathrm{r}}^{\prime} \kappa^{\prime}\right\rangle\left\langle\overrightarrow{\mathrm{r}}^{\prime} \kappa^{\prime} \mid k_{z} \overline{\mathrm{k}} n\right\rangle & \\
= & \epsilon\left(k_{z} \overline{\mathrm{k}} n\right)\left\langle\overrightarrow{\mathrm{r}} \kappa \mid k_{z} \overline{\mathrm{k}} n\right\rangle .
\end{aligned}
$$

Comparison with the usual eigenvalue equation for the normal modes of vibration ${ }^{15}$ shows that

$$
\begin{aligned}
& \epsilon\left(k_{z} \overline{\mathrm{k}} n\right)=\omega^{2}\left(k_{z} \overline{\mathrm{k}} n\right), \\
& \left\langle\overrightarrow{\mathrm{r}} \kappa \mid k_{z} \overline{\mathrm{k}} n\right\rangle=M_{\kappa}^{1 / 2} u\left(\overrightarrow{\mathrm{r}} \kappa, k_{z} \overline{\mathrm{k}} n\right), \\
& \left\langle\overrightarrow{\mathrm{r}} \kappa\left|H_{0}\right| \overrightarrow{\mathrm{r}}^{\prime} \kappa^{\prime}\right\rangle=M_{\kappa}^{-1 / 2} \Phi_{0}\left(\overrightarrow{\mathrm{r}} \kappa, \overrightarrow{\mathrm{r}}^{\prime} \kappa^{\prime}\right) M_{\kappa^{\prime}}^{-1 / 2} .
\end{aligned}
$$

Here $\omega\left(k_{z} \overline{\mathrm{k}} n\right)$ is the frequency for the mode labeled by $k_{z} \overline{\mathrm{k}} n, u\left(\overrightarrow{\mathrm{r}}_{\kappa}, k_{z} \overline{\mathrm{k}} n\right)$ is the properly normalized amplitude of displacement for the atom labeled by $\overrightarrow{\mathbf{r}} \kappa$ when the crystal is vibrating in this mode, $M_{\kappa}$ is the mass of this atom, and $\Phi_{0}\left(\overrightarrow{\mathbf{r}}_{\kappa}, \overrightarrow{\mathbf{r}}^{\prime} \kappa^{\prime}\right)$ is a
$3 \times 3$ matrix whose elements are the force constants coupling the two atoms labeled by $\overrightarrow{\mathbf{r}} \kappa$ and $\vec{r}^{\prime} \kappa^{\prime}$ in the unperturbed crystal. We label the three acoustic modes by $n=1,2$, and 3 . Since all the atoms in the crystal have the same displacement as $\overrightarrow{\mathrm{k}} \rightarrow 0$ for the acoustic modes, as indicated in (2.1.63) of Ref. i5,

$$
u(\overrightarrow{\mathbf{r}} \kappa, 00 n)=A(n), n=1,2 \text {, or } 3,
$$

where $A(n)$ is independent of $\overrightarrow{\mathrm{r}}$ and $\kappa$. Also, $\omega\left(k_{z} \overline{\mathrm{k}} n\right) \propto|\overrightarrow{\mathrm{k}}|$ for the acoustic modes as $\overrightarrow{\mathrm{k}} \rightarrow 0$ along a fixed direction, so for small $k_{z}$

$$
\epsilon\left(k_{z} 0 n\right) \simeq k_{z}^{2} / 2 m_{n}^{*}, \quad n=1,2 \text {, or } 3,
$$

where $m_{n}^{*}$ is a constant. This is of the form (4.2). We thus have three extrema of the kind indicated in (6.1) $-N_{0}=3$-with $\omega_{0}=\overline{\mathrm{k}}=k_{0 i}=0$.

Let

$$
\Delta \Phi\left(\overrightarrow{\mathrm{r}} \kappa, \overrightarrow{\mathrm{r}}^{\prime} \kappa^{\prime}\right) \equiv \Phi\left(\overrightarrow{\mathrm{r}} \kappa, \overrightarrow{\mathrm{r}}^{\prime} \kappa^{\prime}\right)-\Phi_{0}\left(\overrightarrow{\mathrm{r}} \kappa, \overrightarrow{\mathrm{r}}^{\prime} \kappa^{\prime}\right)
$$

where $\Phi$ represents the force constants for the perturbed crystal. According to $(7.2 \mathrm{c})$,

$$
\left\langle\overrightarrow{\mathrm{r}} \kappa|V| \overrightarrow{\mathrm{r}}^{\prime} \kappa^{\prime}\right\rangle=M_{\kappa}^{-1 / 2} \Delta \Phi\left(\overrightarrow{\mathrm{r}} \kappa, \overrightarrow{\mathrm{r}}^{\prime} \kappa^{\prime}\right) M_{\kappa^{\prime}}^{-1 / 2} .
$$

Consider

$$
\begin{aligned}
V\left(k_{z} k_{z}^{\prime} n n^{\prime} \overline{\mathrm{k}}\right) & =\left\langle k_{z} \overline{\mathrm{k}} n\left|\sum_{\overrightarrow{\mathrm{r}} \kappa} \sum_{\overrightarrow{\mathrm{r}}^{\prime} \kappa^{\prime}}\right| \overrightarrow{\mathrm{r}} \kappa\right\rangle\left\langle\overrightarrow{\mathrm{r}}_{\kappa}|V| \overrightarrow{\mathrm{r}}^{\prime} \kappa^{\prime}\right\rangle\left\langle\overrightarrow{\mathrm{r}}^{\prime} \kappa^{\prime}|| k_{z}^{\prime} \overline{\mathrm{k}} n^{\prime}\right\rangle \\
& =\sum_{\overrightarrow{\mathrm{r}} \kappa} \sum_{\overrightarrow{\mathrm{r}}^{\prime} \kappa^{\prime}} u^{*}\left(\overrightarrow{\mathrm{r}}_{\kappa}, k_{z} \overline{\mathrm{k}} n\right) \Delta \Phi\left(\overrightarrow{\mathrm{r}} \kappa, \overrightarrow{\mathrm{r}}^{\prime} \kappa^{\prime}\right) u\left(\overrightarrow{\mathrm{r}}^{\prime} \kappa^{\prime}, k_{z}^{\prime} \overline{\mathrm{k}} n^{\prime}\right) .
\end{aligned}
$$

For $n=1,2$, or $3,(7.3)$ implies that

$V\left(0 k_{z}^{\prime} n n^{\prime} 0\right)$

$$
=A^{*}(n) \sum_{\overrightarrow{\mathrm{r}}^{\prime} \kappa^{\prime}} u\left(\overrightarrow{\mathrm{r}}^{\prime} \kappa^{\prime}, k_{z}^{\prime} \overline{\mathrm{r}} n^{\prime}\right) \sum_{\overrightarrow{\mathrm{r}} \kappa} \Delta \Phi\left(\overrightarrow{\mathrm{r}}_{\kappa}, \overrightarrow{\mathrm{r}}^{\prime} \kappa^{\prime}\right) .
$$

According to (2.1.10) and (2.1.15) of Ref. 15,

$$
\sum_{\overrightarrow{\mathrm{r}} \kappa} \Phi_{0}\left(\overrightarrow{\mathrm{r}}_{\kappa}, \overrightarrow{\mathrm{r}}^{\prime} \kappa^{\prime}\right)=0,
$$

and the same is true of $\Phi$, so

$$
\sum_{\overrightarrow{\mathrm{r}} \kappa} \Delta \Phi\left(\overrightarrow{\mathrm{r}}_{\kappa}, \overrightarrow{\mathrm{r}}^{\prime} \kappa^{\prime}\right)=0 .
$$

This restriction on the change in the force constants makes (7.9) vanish, so

$$
V\left(0 k_{z}^{\prime} n n^{\prime} 0\right)=0 \text { for } n=1,2 \text {, or } 3 \text {. }
$$

Thus three rows of $\tilde{V}(0)$ are equal to zero, or $M_{0}$ $=3$. Then (6.8) implies that

$$
\operatorname{disc} \eta(\omega \overline{\mathrm{k}})=(3-3) \frac{1}{2} \pi=0
$$

at $\overline{\mathrm{k}}=0$, as $\omega$ increases through $\omega_{0}=0$. I.e., there is no discontinuity in the phase shift at $\omega=\overline{\mathrm{k}}=0$ for the acoustic modes, even though they have extrema there.
${ }^{1}$ B. S. DeWitt, Phys. Rev. 103, 1565 (1956).

${ }^{2} \mathrm{G}$. Toulouse, Solid State Commun. 4, 593 (1966).

${ }^{3}$ W. Kohn, Phys. Rev. 115,809 (1959).

${ }^{4}$ W. Kohn, Phys. Rev. B 7, 2285 (1973). The case of a diatomic chain with nearest-neighbor interactions is explicitly treated.

${ }^{5}$ E. I. Blount, Solid State Phys. 13, 305 (1962). See Appendix C.

${ }^{6}$ J. B. Krieger, Phys. Rev. 156, 776 (1967).
${ }^{7} \mathrm{R}$. G. Newton, Scattering Theory of Waves and Particles (McGraw-Hill, New York, 1966). See pp. 332 and 335.

${ }^{8} \mathrm{~J}$. Koutecky, Phys. Rev. 108, 13 (1957).

${ }^{9}$ B. W. Holland, Philos. Mag. 8,87 (1963).

${ }^{10}$ R. A. Brown, Phys. Rev. $15 \overline{6}, 889$ (1967).

${ }^{11} \mathrm{E}$. Hille, Analytic Function Theory, Volume I (Ginn, Boston, 1959), p. 186.

${ }^{12}$ V. Heine, Proc. Phys. Soc. 81,300 (1963). See p. 
304.

${ }^{13}$ See Ref. 7, pp. 438-439.

${ }^{14}$ L. I. Schiff, Quantum Mechanics, 3rd ed. (McGrawHill, New York, 1968), pp. 353-354. Or see pp. 355356 of Ref. 7. Also see p. 311 of Ref. 7.
${ }^{15}$ A. A. Maradudin, E. W. Montroll, G. H. Weiss, and I. P. Ipatova, Theory of Lattice Dynamics in the Harmonic Approximation, 2nd ed. (Academic, New York, 1971). Equation (2.1.46) is the Fourier transformed version of our (7.1). Also see (2.1.9). 